\title{
Vitamin D Treatment and Mortality in Chronic Kidney Disease: A Systematic Review and Meta-Analysis
}

\author{
Flore Duranton $^{a} \quad$ Maria E. Rodriguez-Ortiz ${ }^{c}$ Yohan Duny ${ }^{b}$ Mariano Rodriguez ${ }^{c}$ \\ Jean-Pierre Daurès $^{b}$ Angel Argilés ${ }^{a}$ \\ a RD-Néphrologie and b Laboratoire de Recherche en Biostatistique, Epidémiologie et Recherche Clinique, \\ Institut Universitaire de Recherche Clinique, Montpellier, France; ${ }^{C}$ Unidad de Investigacion, University Hospital \\ Reina Sofia, Cordoba, Spain
}

\section{Key Words}

Chronic kidney disease · Vitamin D · Survival · Mortality ·

Cardiovascular mortality

\begin{abstract}
Background/Aims: Hypovitaminosis D has been associated with an increased cardiovascular mortality in the general population and in patients with chronic kidney disease (CKD). Still, whether prescribing vitamin D reduces the risk of mortality in renal patients remains controversial. Methods: We searched PubMed, ClinicalTrials.gov and the Cochrane Library for long-term longitudinal studies comparing vitamin D compounds (25-hydroxyvitamin D, 1,25-dihydroxyvitamin $\mathrm{D}$ and synthetic derivatives) to placebo or no treatment in renal patients, and which evaluated mortality, to perform a meta-analysis. Data concerning study quality, population and effect size were extracted independently by two investigators using predefined forms. Results: Fourteen observational studies (194,932 patients) met all eligibility criteria. Most studies were performed in hemodialysis patients and all used calcitriol or synthetic analogues. In a random effects meta-analysis, receiving any vitamin $D$ therapy significantly reduced the risk of all-cause mortality (relative risk $0.73,95 \% \mathrm{Cl} 0.65-0.82$ ). The relative risk of death was 0.72 $(95 \% \mathrm{Cl} 0.65-0.80)$ after 3 years of therapy and $0.67(95 \% \mathrm{Cl}$
\end{abstract}

0.45-0.98) after 5 years. In meta-regression, the risk reduction was shown to be greater in patients with higher parathyroid hormone serum levels $(p=0.01)$. The risk of cardiovascular mortality was also significantly reduced in patients receiving any vitamin D derivative (relative risk $0.63,95 \% \mathrm{Cl}$ 0.44-0.92). Conclusion: Therapies with 1,25-dihydroxyvitamin $D$ and analogues are associated with reduced mortality in CKD patients, and particularly in those suffering from secondary hyperparathyroidism. These results, based on observational evidence, are supportive of prescribing vitamin $D$ therapies to CKD patients, while respecting good practice guidelines.

Copyright $\odot 2013$ S. Karger AG, Basel

\section{Introduction}

In the general population as well as in renal patients, low vitamin D precursor levels (25-hydroxyvitamin D: $25(\mathrm{OH}) \mathrm{D})$ are associated with increased risks of cardio-

M.R. and A.A. are members of the European Uremic Toxin working group (EUTox) of the European Society for Artificial Organs endorsed by the European Renal Association - European Dialysis and Transplant Association.

\section{KARGER}

E-Mail karger@karger.com

www.karger.com/ajn
Àngel Argilés

RD-Néphrologie

104 rue de la Galéra

FR-34090 Montpellier (France)

E-Mail argiles@rd-n.org 
vascular events and death [1-3]. The active form of vitamin $\mathrm{D}, 1,25$-dihydroxyvitamin $\mathrm{D}_{3}\left(1,25(\mathrm{OH})_{2} \mathrm{D}\right)$, is a hormone classically known for regulating bone and mineral homeostasis, but additional biological effects including endothelial and cardiovascular protection, immunomodulation and antitumoral activities have recently been observed [4]. In the general population, restoring $25(\mathrm{OH})$ $\mathrm{D}$ levels with nutritional supplementation reduced the risk of mortality [5], but it is not known whether this effect remains in patients with chronic kidney disease (CKD). Vitamin D insufficiency, defined as 25(OH)D serum levels $<30 \mathrm{ng} / \mathrm{ml}$, affects up to $75 \%$ of CKD patients and can be corrected by nutritional vitamin $\mathrm{D}$ [6-8]. However, the impaired $1,25(\mathrm{OH})_{2} \mathrm{D}$ synthesis due to the reduced availability of the renal enzyme 1- $\alpha$-hydroxylase could preclude biological activities. Still, an abundant body of evidence shows that treatments with vitamin $\mathrm{D}$ derivatives (natural or synthetic $1,25(\mathrm{OH})_{2} \mathrm{D}$ or synthetic prohormones) ameliorate mineral and bone disorders observed in CKD and improve anemia in dialysis patients $[9,10]$. A previous meta-analysis of the putative benefits of treatments with these vitamin $\mathrm{D}$ derivatives in renal patients demonstrated that they had a proven efficacy in reducing serum alkaline phosphatase and parathyroid hormone (PTH) levels but did not influence survival [11]. This is in contrast with the clinical impression of a favorable evolution of treated patients, which is also supported by epidemiological observations in hemodialysis patients $[12,13]$. Direct and indirect effects of $1,25(\mathrm{OH})_{2} \mathrm{D}$ on the cardiorenal system are likely to occur at early stages of the disease $[14,15]$, suggesting that early restoration of its activity could delay dialysis initiation or death. Because the limited number of randomized controlled trials (RCTs) estimating the effect of vitamin D therapies on survival of CKD patients led to inconclusive results in a previous analysis [11], we decided to further examine this question in a meta-analysis including RCTs as well as longitudinal observational studies. We carried out a meta-analysis to evaluate the association between the use of any kind of vitamin D therapy and the risk of all-cause and cardiovascular mortality in patients affected by CKD who were followed during an average period above 6 months.

\section{Material and Methods}

\section{Study Search and Selection}

On September 1, 2010, PubMed, ClinicalTrials.gov and the Cochrane Library were searched for articles combining terms related to vitamin D (e.g. vitamin D, cholecalciferol, calcipotriol), CKD (e.g. kidney diseases, renal replacement therapy, ESRD), and mortality or cardio-vascular outcome (e.g. mortality, survival rate, coronary risk), with no time or language restrictions. Because no results met the search criteria in the ClinicalTrials.gov database, outcome terms were removed from this search (online suppl. table S1; see www.karger.com/doi/10.1159/000346846 for all online suppl. material). Authors were contacted to retrieve full-text articles when not available otherwise. Unpublished abstracts presented during the ERA-EDTA Congress (Munich, Germany, 2010) and the ASN Renal Week (Denver, Colo., USA, 2010) and literature citations were hand searched for additional studies. Reporting methods were adapted from MOOSE and PRISMA guidelines for meta-analyses $[16,17]$.

Studies were included in the meta-analysis if they matched all pre-specified eligibility criteria. Articles had to be original studies comparing vitamin $\mathrm{D}$ use to receiving a placebo or no treatment. Additional inclusion criteria were: (1) exclusion of kidney transplant patients; (2) minimal follow-up of 6 months; (3) occurrence of at least 1 death per treatment group, and (4) sufficient data to determine the relative risk and confidence interval (CI) of all-cause or cardiovascular mortality between vitamin $\mathrm{D}$-treated and vitamin D-untreated patients. Searches, study selection and data extraction were performed independently by two investigators from different institutions (F.D. and M.E.R.-O.). Discrepancies were solved by discussion until consensus.

\section{Data Extraction and Quality Assessment}

From eligible studies, two reviewers independently extracted data using piloted forms (F.D. and M.E.R.-O.). The outcomes of interest were all-cause mortality, cardiovascular mortality and 3and 5-year all-cause mortality. From each study, relative risks and 95\% CI were extracted or estimated from computed estimates such as hazard ratios or from sample sizes and death rates per group [18]. In predialysis studies, deaths occurring during predialysis and dialysis stages were considered. When results were stratified, the largest stratum was included. To evaluate study quality, information depicting trial characteristics and baseline demographic and biological characteristics of patients were extracted. Because our search resulted in observational studies only, study quality was evaluated based on study design, assessment of confounding and adequacy of statistical adjustments.

\section{Statistical Analysis}

We evaluated the effect of vitamin D therapy on mortality from any cause and from cardiovascular causes, and on the 3- and 5-year all-cause mortality. Results were expressed as relative risks (RRs), defined as the ratio of the mortality rate in patients receiving vitamin $\mathrm{D}$ therapy over the mortality rate of patients not receiving vitamin $\mathrm{D}$. Relative risks $<1$ suggest a protective effect of therapy. Study-specific RRs were pooled under random-effects models using the DerSimonian-Laird approach to account for expected heterogeneity [19]. Heterogeneity was assessed using the $I^{2}$ statistic for which values $>50 \%$ may indicate substantial heterogeneity. The influence of sample, treatment and methodological parameters on RRs was assessed by subgroup analyses and logarithmic mixedeffects meta-regressions. The influence of within-study differences in demographic and biological characteristics of treated and control groups was tested using the same approaches. The risk of publication bias was assessed by one-tailed Egger's test, by funnel plot and by Duval and Tweedie's trim and fill method [20,21]. Orwin's 
Fig. 1. Flow diagram of the selection pro-

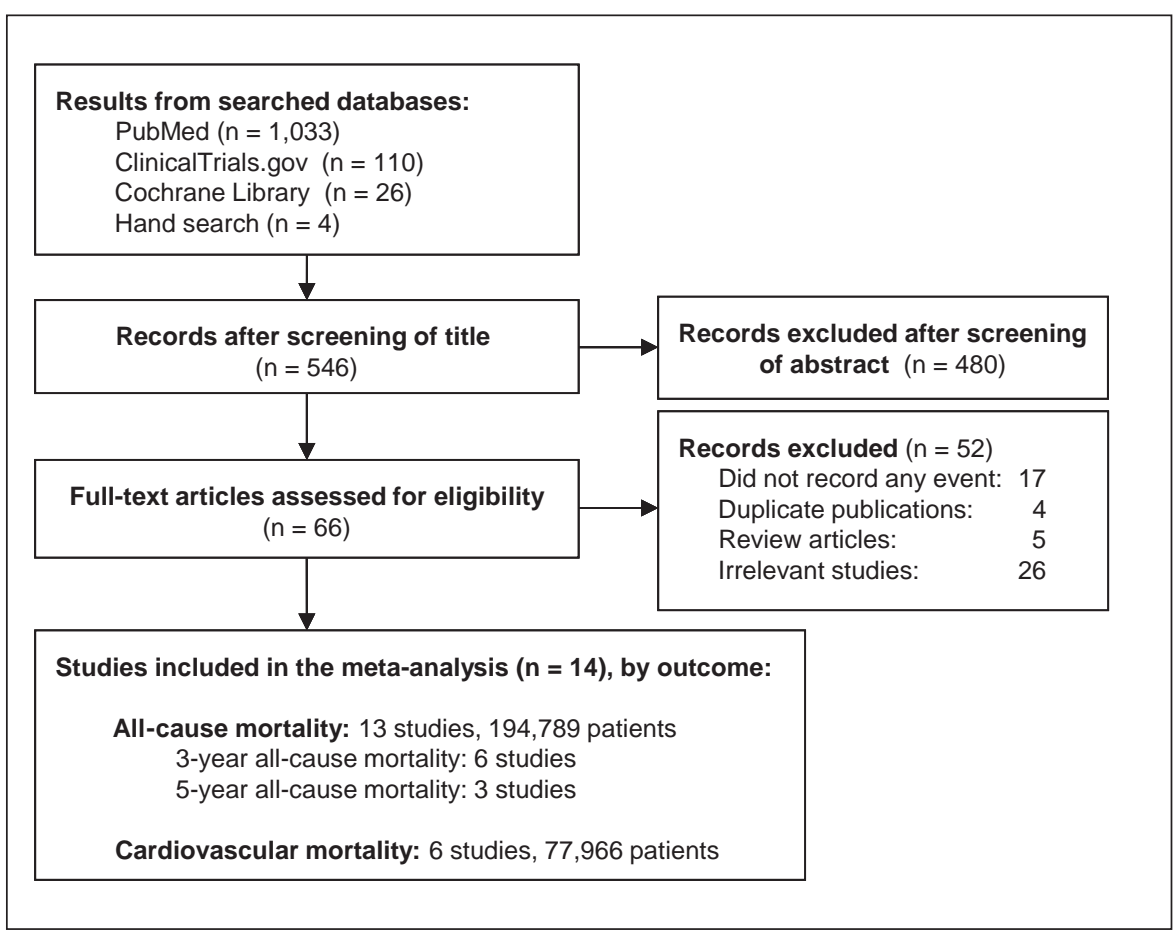

fail-safe $\mathrm{N}$ was calculated to estimate the number of additional non-significant studies necessary to make the pooled result of the meta-analysis reach a predefined critical value [22]. Statistical analyses were performed using Comprehensive Meta-Analysis, version 2 (Biostat Inc., Englewood, N.J., USA) and SAS, version 9.2 (SAS Institute Inc., Cary, N.C., USA).

\section{Results}

\section{Search Results}

A total of 1,169 records were identified through electronic databases PubMed, ClinicalTrial.org and the Cochrane Library, and 4 records were identified by hand (fig. 1). After screening of titles and removal of reviews, duplicate publications and irrelevant research, there remained 546 records. Screening of abstracts led to 66 remaining records of which 52 were excluded after full-text analysis because they were reviews, publications on the same study (e.g. [23]), did not provide sufficient data on effect sizes for any studied outcome (e.g. [24, 25]) or were irrelevant to the question. Consequently, 14 records were included in the meta-analysis (table 1). They included an abstract [36] and an e-published article [26], both identified through the Renal Week Symposium 2010 abstract book. An additional publication [28] providing complementary information about the original study [27] con- tributed to the analysis but was considered as the same work.

Records were prospective (7 studies) or retrospective (7 studies) observational studies (table 1), and there was no blinding or randomization of patients in any studies. Overall, a total of 194,932 patients were followed over an average duration of $4.5 \pm 3.6$ years. When given, loss to follow-up was $<12 \%[29,32-34]$. There were 3 studies conducted in predialysis CKD patients $[29,33,35]$. Four studies were performed in patients incident to hemodialysis $[31,37,38,40]$ and the remaining 7 studies in patients already on hemodialysis $[26,27,30,32,34$, 36, 39]. Administered molecules were calcitriol (natural $1,25(\mathrm{OH})_{2} \mathrm{D}_{3}$ ), paricalcitol (synthetic $1,25(\mathrm{OH})_{2} \mathrm{D}_{3}$ analogue), alfacalcidol (synthetic prohormone, $\left.1 \alpha(\mathrm{OH}) \mathrm{D}_{3}\right)$ or doxercalciferol (synthetic prohormone, $1 \alpha(\mathrm{OH}) \mathrm{D}_{2}$ ). No study evaluated interventions with $25(\mathrm{OH}) \mathrm{D}$ supplementation or dietary precursors. One study evaluated the effect of 'active Vitamin D' but did not specify which compounds were included [36]. Treatments were given orally or by intravenous injection; 1 study did not detail the administration route [30]. Exposure was defined as receiving any dose of a vitamin D compound during follow-up.

Baseline characteristics of patients according to treatment group were available in 11 studies and absent from 


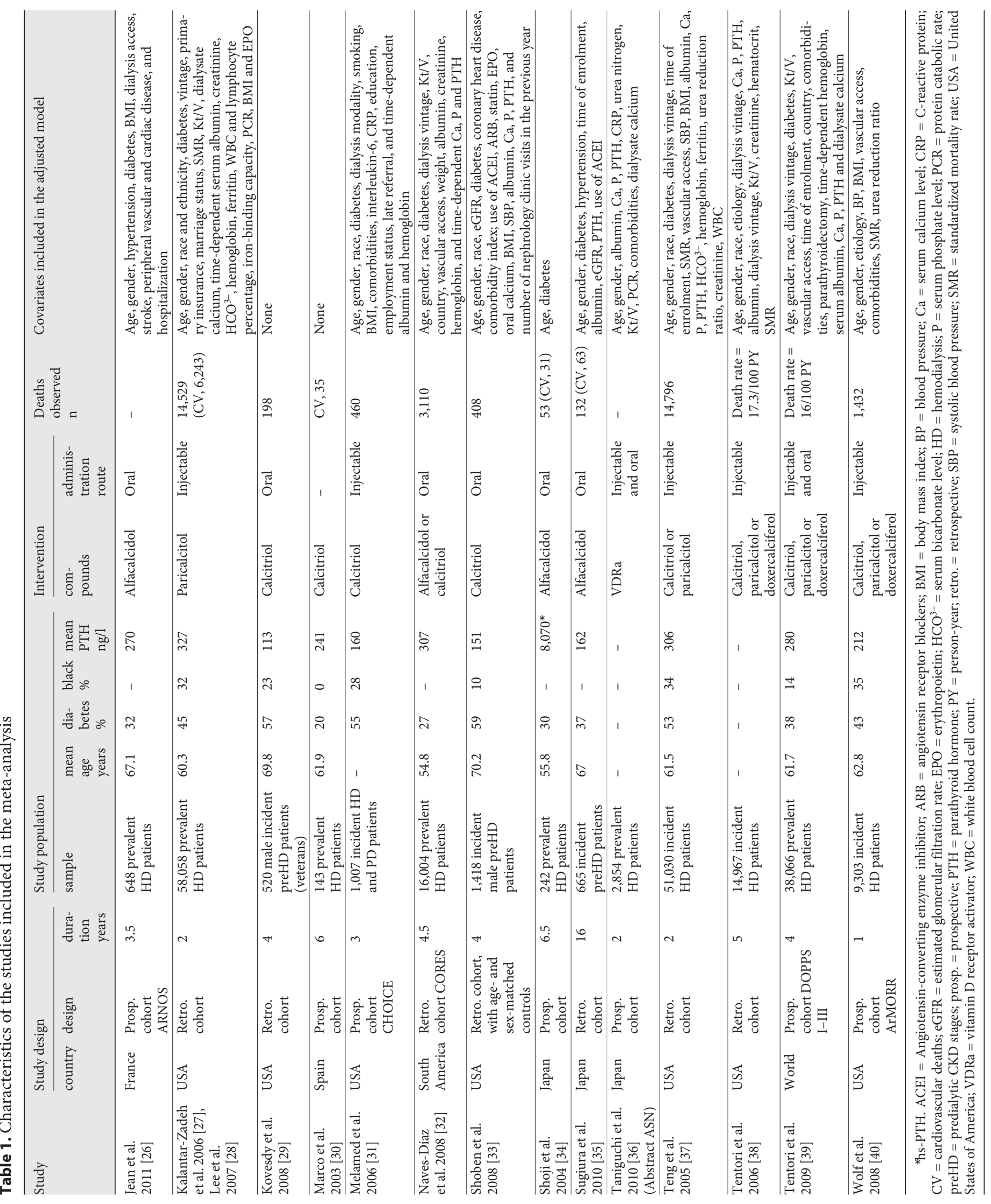




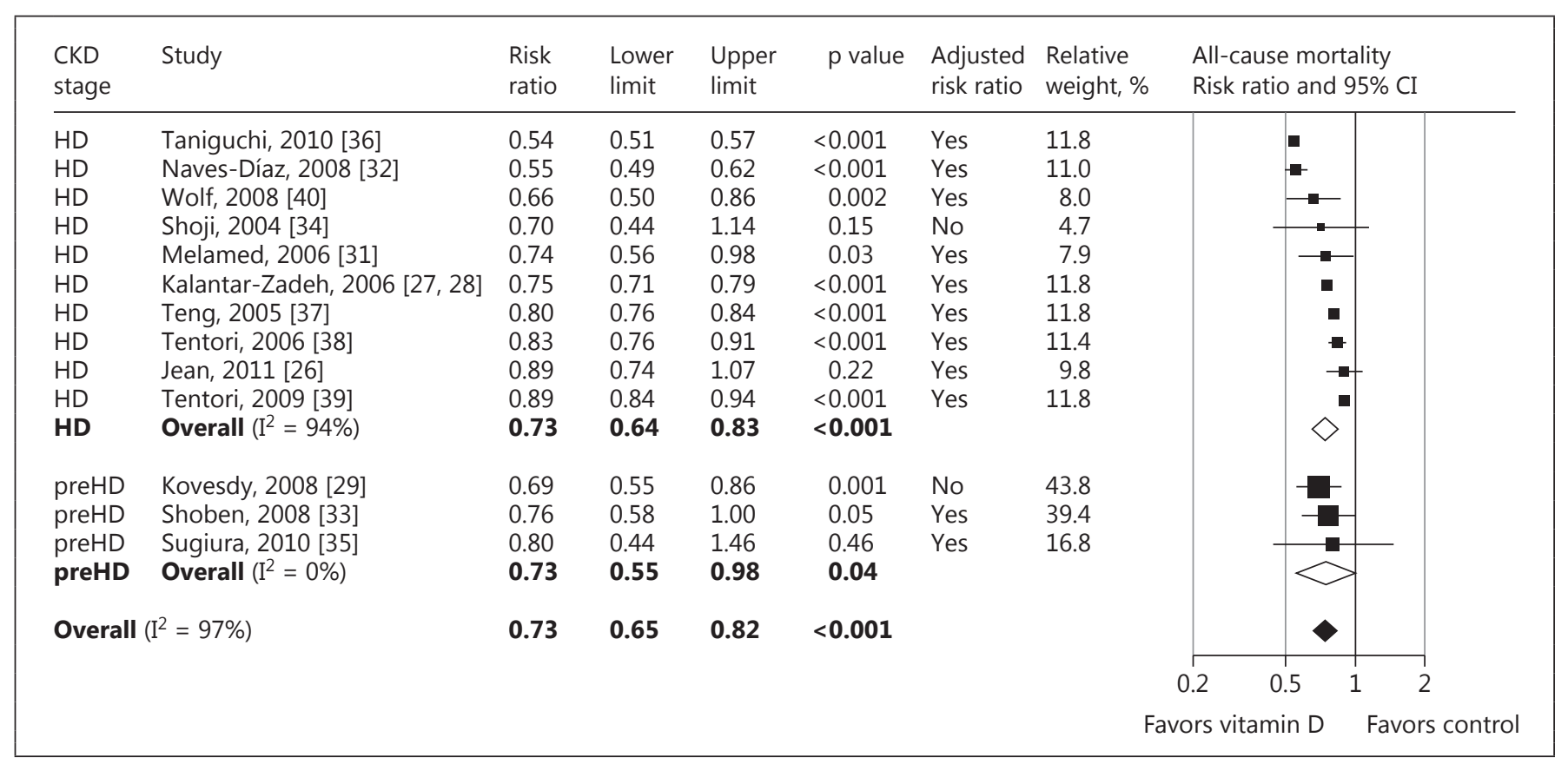

Fig. 2. Forest plots and summary estimates of all-cause mortality RRs depending on vitamin $D$ treatment in hemodialysis patients (HD) or patients at CKD stages not requiring dialysis (preHD). RRs $<1$ indicate a greater chance of survival in the vitamin D therapy group as compared with the control group.

3 studies [30, 31, 36]. There were slight but consistent differences between treatment groups. Treated patients had greater baseline PTH levels $(253 \pm 121$ vs. $159 \pm 82 \mathrm{ng} / \mathrm{l}$, $\mathrm{p}=0.002)$ and serum creatinine levels $(6.9 \pm 3.3$ vs. $6.5 \pm$ $3.0 \mathrm{mg} / \mathrm{dl}, \mathrm{p}=0.03)$ and were slightly younger $(62.4 \pm 5.4$ vs. $64.1 \pm 5.3$ years, $\mathrm{p}=0.03)$. Four studies reported information on parathyroidectomy. At baseline, its prevalence was $<9 \%$ and was not influenced by treatment group [26, $34,38]$. During follow-up, the incidence of parathyroidectomy was $<0.15 \%$ [37].

Statistical adjustments were frequently applied to control for demographical, biological and therapeutic parameters and showed a large range of adjusted parameters (table 1). Based on study design, assessment of confounding and adequacy of statistical adjustments, the quality of included studies ranged from rather low to satisfactory (online suppl. table S2). The most frequently observed limitations concerned the comparability of the treated and untreated populations, and the population selection following the use of statistical models adjusting for many variables. The crude and adjusted RRs which were used in the meta-analysis are available in online suppl. table S3.

Vitamin D Therapy and Mortality in CKD

\section{All-Cause Mortality}

The association of vitamin $\mathrm{D}$ therapy with all-cause mortality was assessed in 13 studies [26-29, 31-40] (fig. 2). Ten studies reported a significant inverse association between receiving vitamin $\mathrm{D}$ and the risk of death. The pooled result showed that receiving vitamin $\mathrm{D}$ was significantly associated with a $27 \%$ relative risk reduction of all-cause mortality (relative risk 0.73 , 95\% CI $0.65-$ 0.82 ). The beneficial effect was equally present among hemodialyzed patients and predialytic patients. Heterogeneity was high $\left(\mathrm{I}^{2}>50 \%\right)$ in the overall meta-analysis and among studies performed in hemodialysis patients. It was absent from the subgroup of studies performed in predialysis patients $\left(\mathrm{I}^{2}=0 \%\right)$. There were two study-specific estimates which did not include any adjustments [29,34]; excluding these two crude results did not influence the results (table 2). Pooling the 11 crude RRs which were available increased the relative risk reduction to $35 \%$ (table 2).

The association between vitamin $\mathrm{D}$ therapy and the risk of death after 3 and 5 years of follow-up could be extracted from 6 and 3 studies, respectively (fig. 3). Most estimates were crude associations derived from survival 


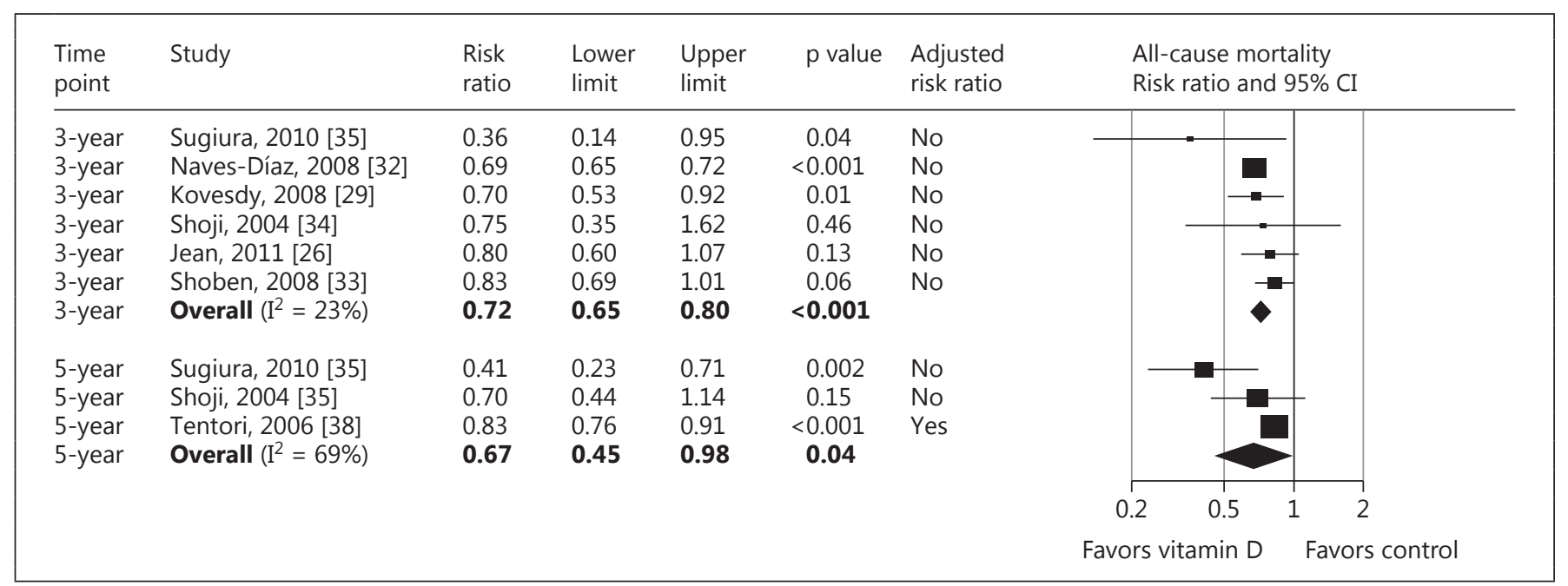

Fig. 3. Forest plots and summary estimates of all-cause mortality RRs depending on vitamin D treatment after 3 years of follow-up (3-year) or 5 years of follow-up (5-year).

Table 2. Summary estimates from random-effects meta-analyses on adjusted and unadjusted relative risks

\begin{tabular}{|c|c|c|c|c|c|c|}
\hline \multirow[t]{2}{*}{ Outcome } & \multirow[t]{2}{*}{ Studies, $\mathrm{n}$} & \multicolumn{3}{|c|}{ Effect size and 95\% CI } & \multirow[t]{2}{*}{$\mathrm{p}$ value } & \multirow[t]{2}{*}{$\mathrm{I}^{2}$} \\
\hline & & RR estimate & lower limit & upper limit & & \\
\hline \multicolumn{7}{|l|}{ All-cause mortality } \\
\hline Adjusted RR & 11 & 0.73 & 0.64 & 0.83 & $<0.001$ & $95 \%$ \\
\hline Unadjusted RR & 11 & 0.65 & 0.58 & 0.73 & $<0.001$ & $94 \%$ \\
\hline \multicolumn{7}{|c|}{ Cardiovascular mortality } \\
\hline Adjusted RR & 4 & 0.55 & 0.41 & 0.74 & $<0.001$ & $63 \%$ \\
\hline Unadjusted RR & 4 & 0.53 & 0.27 & 1.06 & 0.069 & $83 \%$ \\
\hline
\end{tabular}

curves. After 3 years of follow-up, vitamin D therapy was significantly associated with a $28 \%$ relative risk reduction in mortality (RR $0.72,95 \%$ CI $0.65-0.80$ ), with a limited heterogeneity. Five years after study initiation, the association was similar (RR 0.67, 95\% CI 0.45-0.98), with slightly greater heterogeneity.

\section{Cardiovascular Mortality}

The association of vitamin D therapy and cardiovascular mortality was assessed in 6 studies $[27,30,32,34-$ 36] (fig. 4). Receiving vitamin $D$ was significantly associated with a $37 \%$ relative reduction of cardiovascular mortality risk (RR 0.63 , 95\% CI 0.44-0.92). Two studies reported crude associations [27,30]. Limiting the analysis to the 4 statistically adjusted RRs, the effect of vitamin D compounds increased to a significant $45 \%$ relative risk reduction of cardiovascular mortality (RR $0.55,95 \%$ CI
$0.41-0.74$ ) and reduced heterogeneity (table 2). Unadjusted RR led to a similar pooled effect which was no longer significant.

\section{Heterogeneity and Publication Bias}

A significant association was observed between studyspecific relative risks of death and hyperparathyroidism in treated patients $(\mathrm{p}=0.011)$. Compared to untreated patients, the higher the baseline PTH levels in the treated group, the stronger the relative risk reduction in mortality (fig. 5). This was modeled by mixed-effects meta-regression on the difference between average PTH levels in treatment and control groups at baseline. The intercept of the regression line was 0.92 (95\% CI 0.74-1.14) and for each differential increase of $100 \mathrm{ng} / \mathrm{l}$ of PTH levels between groups, the relative risk of death significantly decreased by $16 \%$ (RR $0.84,95 \%$ CI $0.73-0.96$ ). None of the 


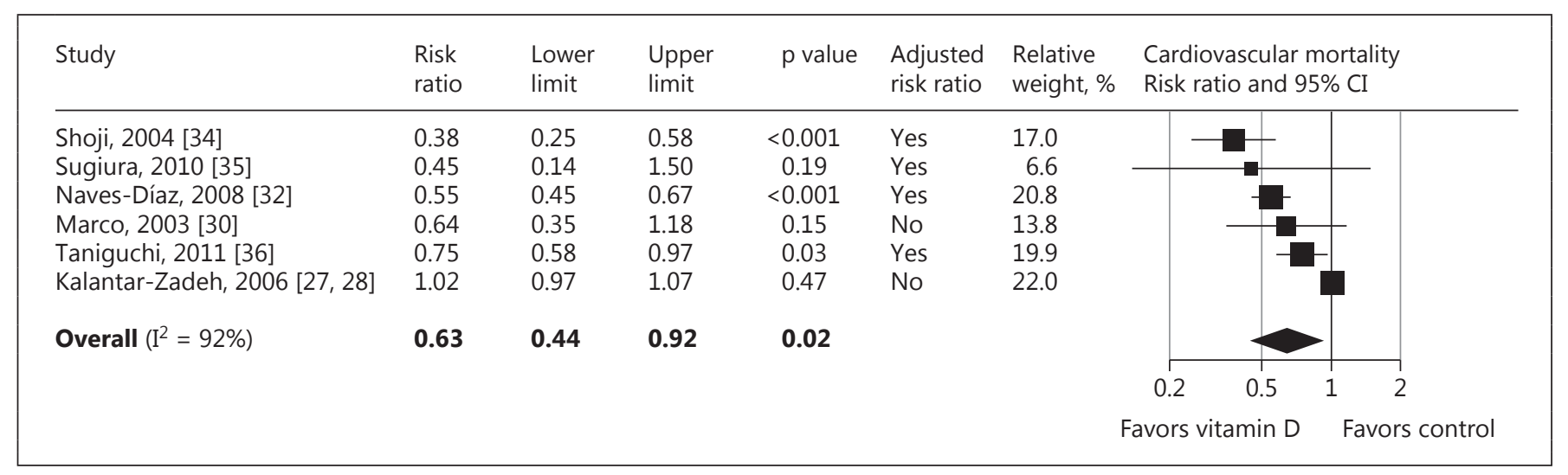

Fig. 4. Forest plot and summary estimate of cardiovascular mortality RRs depending on vitamin $\mathrm{D}$ treatment.

Fig. 5. Relationship between the difference in PTH levels between treatment groups and the relative risk of all-cause mortality. For each study, the difference in PTH levels was calculated at baseline, as the mean $\mathrm{PTH}$ level in the treated group minus the mean PTH level in the untreated group. A log linear model was fitted to the data, weighting each study by the inverse of its variance (black line). Each 100-pg/ml relative increase of PTH levels in the vitamin D group was associated with a $17 \%$ reduction of the relative mortality.

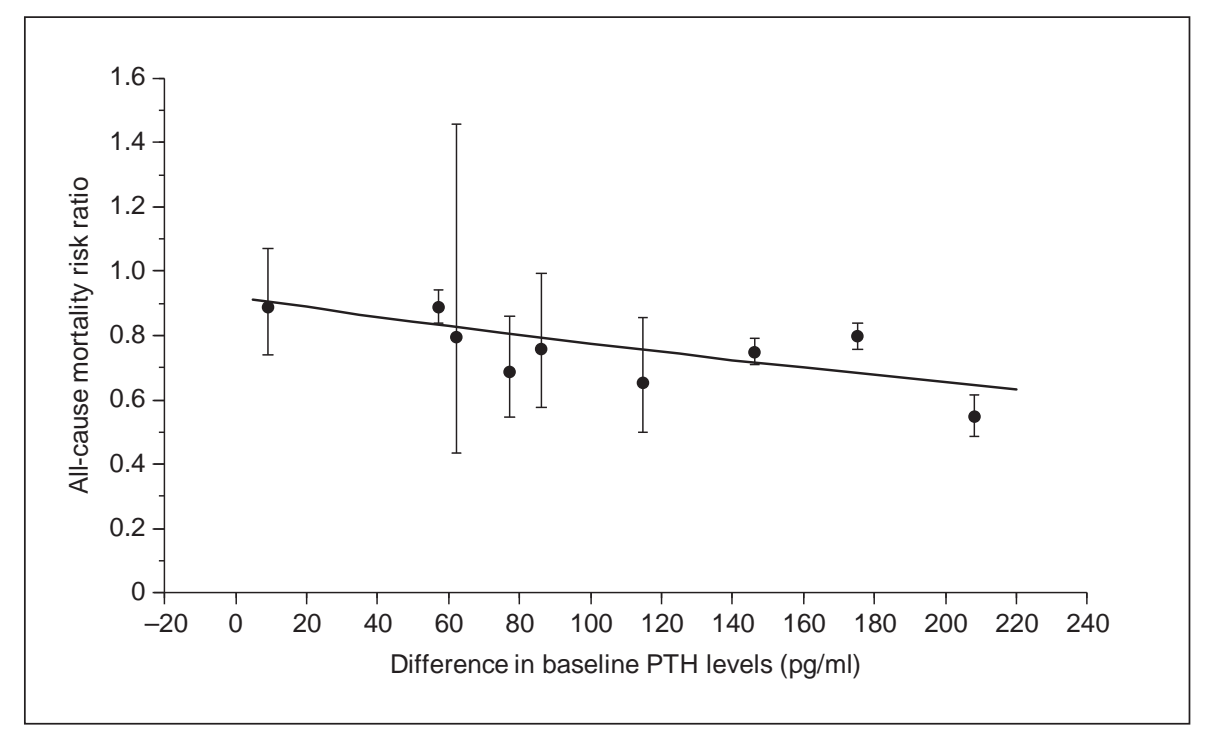

other clinical parameters which were tested (age, diabetes prevalence, albumin or creatinine levels) significantly influenced mortality RRs ( $\mathrm{p}>0.05$ ).

The sample, intervention and methodological characteristics of the studies which were tested did not significantly influence results concerning all-cause mortality ( $p>0.05$, online suppl. table S4). In prevalent hemodialysis patients (6 studies) and in patients incident to hemodialysis (4 studies), the effects of vitamin $\mathrm{D}$ derivatives on all-cause mortality were 0.70 (95\% CI 0.59-0.84) and 0.76 (95\% CI 0.67-0.96), respectively. Pooled estimates from the 6 prospective studies and the 7 retrospective studies were nearly identical $(0.73,95 \%$ CI $0.60-0.88$ and 0.73 , 95\% CI 0.61-0.87, respectively).

Vitamin D Therapy and Mortality in CKD
In one-study removed analyses, pooled estimates of all-cause mortality RR varied in a narrow range, from 0.71 (95\% CI $0.63-0.81$ ) to 0.76 (95\% CI $0.70-0.82$ ), showing the limited influence of single studies on the overall estimate (online suppl. table S5). Egger's test showed no evidence of publication bias of studies on allcause mortality RR $(\mathrm{p}=0.45)$. This was consistent with the symmetrical shape of the funnel plot (online suppl. fig. S1). Adding the virtually missing study from the trim and fill method did not influence pooled results. Finally, according to Orwin's fail-safe N, it would take 26 null studies to add to find an overall effect equal to a $10 \%$ relative risk reduction, signifying that unpublished non-significant studies would affect our results in a limited manner. 


\section{Discussion}

Our meta-analysis found a significant 27\% lower mortality risk in CKD patients receiving vitamin $\mathrm{D}$ therapies in the form of alfacalcidol, calcitriol or analogues. The relative risk reduction was greater with longer follow-up and for deaths attributable to cardiovascular causes $(37 \%$ reduction). These results support the clinical impressions of renal physicians in favor of administering these types of vitamin D derivatives to CKD patients for their expected benefits. The survival advantage was equally observed in patients at early stages of CKD and in patients undergoing dialysis and the risk reduction was greater when treated patients were more severely affected by hyperparathyroidism.

To our knowledge, only 1 previous meta-analysis on vitamin $\mathrm{D}$ treatments provided results on mortality in CKD and hemodialysis patients [11]. This study included exclusively RCTs and found no influence of vitamin D therapy on mortality. However, this result, contrary to the general feeling of the renal community, needs to be carefully interpreted as it may be influenced by the lack of controlled trials analyzing an effect of vitamin $\mathrm{D}$ derivatives in cases of severe deficiency, since in these cases a placebo administration would have been unethical [41]. Furthermore, this estimation was based on a small number of RCTs which did not study mortality as the main objective. Altogether, these trials observed a limited number of deaths (16 deaths) occurring in 509 patients [11,42, 43]. Our selection criteria included a minimal follow-up of 6 months to ensure sufficient time for the drug effect to occur and the outcome to be observed. Furthermore, they were settled to identify studies focusing on the association between any type of vitamin $\mathrm{D}$ therapy and mortality or cardiovascular diseases, in order to gather appropriately estimated results on the outcomes of interest. These stringent selection criteria failed to retain RCTs, as no RCT fulfilled them, but permitted the inclusion of 14 relevant epidemiological studies, including several large ones, observing altogether a very large number of cases ( $>35,155$ deaths) in a total population of 194,932 patients with CKD or undergoing dialysis. The present work is, therefore, the first meta-analysis conducted to specifically estimate the treatment effect of any type of vitamin D derivative on the hard outcomes that are all-cause and cardiovascular mortality in CKD.

Overall, the majority of patients included in our analysis were clinically representative of stage $5 \mathrm{CKD}$ patients, and they benefited from vitamin $\mathrm{D}$ therapy when they received it. However, a greater effect was observed in vi- tamin D-deficient patients $[26,36]$. The high prevalence of vitamin D insufficiency and deficiency in CKD patients at early and final stages [7] suggests that many patients could benefit from nutritionally or medically restoring vitamin $\mathrm{D}$ activity. We showed that patients with greater alterations of bone and mineral homeostasis as indicated by increased serum PTH levels benefited even more from the therapy. This is interesting as serum PTH has been independently associated with increased mortality [23, $27,44]$. One could think that in our analyses treated patients, who generally had greater baseline PTH levels than untreated patients, would be at increased risk of dying. However, the proven effect of $1,25(\mathrm{OH})_{2} \mathrm{D}$ on reducing hyperparathyroidism [45] did not only reduce a supposedly increased relative risk due to elevated PTH levels to 1 , but reduced the RRs to values $<1$, suggesting the existence of PTH-independent biological activities of $1,25(\mathrm{OH})_{2} \mathrm{D}$. The latter effects could also be responsible for the observed $8 \%$ risk reduction after vitamin $\mathrm{D}$ therapy which was present, although not statistically significant, when treated and untreated patients had similar baseline PTH levels.

In epidemiological studies, the dose-response pattern of vitamin D derivatives tended to show reduced benefits at the greater doses $[27,32]$. When the mean daily intake of calcitriol or alfacalcidol exceeded $1 \mu \mathrm{g}$, therapies were no longer associated with survival benefits [32]. At inappropriately high doses of calcitriol or analogues, adverse effects such as hypercalcemia could overcome its protective effects. Alternatively, it could also be that the high doses were still insufficient for patients with greatly elevated PTH levels. This hypothesis is supported by the dose-response effect which appears after adjusting doses to PTH levels, showing that receiving higher doses reduced the risk of mortality to a greater extent [23]. Patients receiving greater doses are probably combined into a heterogeneous group in which both situations occur. Monitoring changes in $25(\mathrm{OH}) \mathrm{D}$ and $1,25(\mathrm{OH})_{2} \mathrm{D}$ serum levels after vitamin $\mathrm{D}$ therapy could have helped understanding the physiopathological ways involved in the risk reduction, but requiring this information would have been an excessively restrictive selection criteria. Confirming our expectations, a recent analysis failed to retrieve studies on vitamin $\mathrm{D}$ supplementation evaluating both mortality and changes in $25(\mathrm{OH}) \mathrm{D}$ serum levels in CKD patients [8].

One limitation of the present work could be the large heterogeneity which was observed across the different study results on mortality. Because we hypothesized a clinical heterogeneity between different CKD stages, a 
stratified analysis was performed according to the use of renal replacement therapy. Interestingly, heterogeneity was absent across results from non-dialyzed patients. Furthermore, therapy significantly reduced mortality to the same extent in dialyzed or in non-dialyzed patients. The fact that results were consistent independently of heterogeneity is comforting and legitimates their interpretation. Another possible limitation of our work is the inclusion of epidemiological evidence, which is prone to be affected by bias and unmeasured confounding, which would therefore remain in our meta-analysis. However, it is unlikely that the observed effect was solely due to survival bias as results remained similar after stratification for incident and prevalent populations. Confounding by indication might have occurred as we found small but systematic differences in clinical characteristics between treated and control groups which were consistent with indications for receiving vitamin $\mathrm{D}$ derivatives (elevated serum PTH and creatinine levels). To estimate the effect of therapy with vitamin $\mathrm{D}$ derivatives, independently of other patient characteristics, we used adjusted relative risks in the meta-analysis as recommended [46]. Overadjustment, which occurs after adjusting for a covariate involved in the causal pathway of the studied effect, might have been present at the study level. Still, it generally tends to underestimate the effect [47]. Disparities in concomitant medications might also have been a source of confounding. Since their introduction in 2004, calcimimetics have been used alone or in association with vitamin D derivatives in order to reduce serum PTH and calcium levels. However, the follow-up periods of most of the included studies were anterior to this date. It is therefore unlikely that calcimimetics were responsible for the observed effects. Furthermore, a recent trial suggests no direct effect of calcimimetics on survival [48].

One-study removed analysis, Egger's tests, Orwin's fail-safe $\mathrm{N}$ and the funnel plot showed a low risk of publication bias. However, we cannot exclude the possibility that non-significant results were not reported, in particular in the context of pharmacoepidemiology where con- flicts of interest are likely to be present. Relationships with the industry were present in 8 of the included studies in the form of industrial partnership or funding, and exhaustiveness of disclosure cannot be assured. We are aware that our results are unlikely to be identically reproduced in an RCT. However, they clearly show a significant association between the use of vitamin $\mathrm{D}$ derivatives and a lower death risk in CKD patients.

A recent placebo-controlled RCT on paricalcitol was performed in $>200$ mild to moderate CKD patients [49]. Receiving paricalcitol did not improve intermediate cardiac endpoints, but was associated with fewer cardiovascular-related hospitalizations. Unfortunately for our question, no deaths occurred during a follow-up lasting over 1 year. While results from well-designed randomized clinical trials addressing the effect of vitamin $\mathrm{D}$ compounds on $25(\mathrm{OH}) \mathrm{D}$ status and mortality or dialysis initiation are awaited, available epidemiological evidence consistently showed that patients receiving vitamin D treatments were at lower risk of mortality. Our results are supportive of prescribing vitamin $\mathrm{D}$ derivatives to $\mathrm{CKD}$ patients, as widely accepted in the clinical community. This is particularly adapted to those patients with elevated PTH levels, provided that good practice is respected. The side effects of vitamin $\mathrm{D}$ derivatives should be assessed and prevented. In this regard, clinicians should adapt their practice with respect to serum calcium and phosphate levels to comply with the guidelines for bone and mineral metabolism in CKD and avoid hypercalcemia and hyperphosphatemia.

\section{Disclosure Statement}

M.R. and M.E.R.-O. received research support from EU Funding through SysKid (FP7-241544).

M.R. has received research grants from Amgen and Fresenius Medical Care and has given lectures for Amgen, Abbott, Shire and Fresenius Medical Care in the previous 36 months. The other authors have no conflicts of interest to declare.

\section{References}

1 Wang TJ, Pencina MJ, Booth SL, et al: Vitamin D deficiency and risk of cardiovascular disease. Circulation 2008;117:503-511.

$\checkmark 2$ Melamed ML, Michos ED, Post W, Astor B: 25-hydroxyvitamin $D$ levels and the risk of mortality in the general population. Arch Intern Med 2008;168:1629-1630.

Vitamin D Therapy and Mortality in CKD
3 Pilz S, Iodice S, Zittermann A, Grant WB, Gandini S: Vitamin D status and mortality risk in CKD: a meta-analysis of prospective studies. Am J Kidney Dis 2011;58:374-382.

4 Rojas-Rivera J, De La Piedra C, Ramos A, Ortiz A, Egido J: The expanding spectrum of biological actions of vitamin D. Nephrol Dial Transplant 2010;25:2850-2865.
5 Autier P, Gandini S: Vitamin D supplementation and total mortality: a meta-analysis of randomized controlled trials. Arch Intern Med 2007;167:1730-1737.

6 Massry S: K DOQI Clinical Practice Guidelines for bone metabolism and disease in chronic kidney disease. Am J Kidney Dis 2003;42:S16. 
7 Ravani P, Malberti F, Tripepi G, et al: Vitamin $\mathrm{D}$ levels and patient outcome in chronic kidney disease. Kidney Int 2009;75:88-95.

-8 Kandula P, Dobre M, Schold JD, et al: Vitamin $\mathrm{D}$ supplementation in chronic kidney disease: a systematic review and meta-analysis of observational studies and randomized controlled trials. Clin J Am Soc Nephrol 2011;6: 50-62.

$\checkmark 9$ Argilés A, Lorho R, Mourad G, Mion CM: High-dose alfacalcidol for anaemia in dialysis. Lancet 1993;342:378-379.

10 Argilés A, Mourad G, Lorho R, et al: Medical treatment of severe hyperparathyroidism and its influence on anaemia in end-stage renal failure. Nephrol Dial Transplant 1994;9: 1809-1812.

-11 Palmer SC, McGregor DO, Macaskill P, et al: Meta-analysis: vitamin D compounds in chronic kidney disease. Ann Intern Med 2007;147:840-853.

12 Park CW, Oh YS, Shin YS, et al: Intravenous calcitriol regresses myocardial hypertrophy in hemodialysis patients with secondary hyperparathyroidism. Am J Kidney Dis 1999;33: 73-81.

13 Wolf M, Shah A, Gutierrez O, et al: Vitamin $\mathrm{D}$ levels and early mortality among incident hemodialysis patients. Kidney Int 2007;72: 1004-1013.

$\checkmark 14$ Rostand SG, Drüeke TB: Parathyroid hormone, vitamin $\mathrm{D}$, and cardiovascular disease in chronic renal failure. Kidney Int 1999;56: 383-392.

-15 Tian J, Liu Y, Williams LA, de Zeeuw D: Potential role of active vitamin $\mathrm{D}$ in retarding the progression of chronic kidney disease. Nephrol Dial Transplant 2007;22:321-328.

-16 Stroup DF, Berlin JA, Morton SC, Olkin I, Williamson GD, Rennie D, Moher D, Becker BJ, Sipe TA, Thacker SB: Meta-analysis of observational studies in epidemiology: a proposal for reporting. Meta-analysis Of Observational Studies in Epidemiology (MOOSE) group. JAMA 2000;283:2008-2012.

17 Moher D, Liberati A, Tetzlaff J, Altman DG: Preferred reporting items for systematic reviews and meta-analyses: the PRISMA statement. PLoS Med 2009;6:e1000097.

$\checkmark 18$ Parmar MKB, Torri V, Stewart L: Extracting summary statistics to perform meta-analyses of the published literature for survival endpoints. Stat Med 1998;17:2815-2834.

$\checkmark 19$ DerSimonian R, Laird N: Meta-analysis in clinical trials. Control Clin Trials 1986;7:177188.

-20 Egger M, Davey Smith G, Schneider M, Minder C: Bias in meta-analysis detected by a simple, graphical test. Brit Med J 1997;315: 629-634.

21 Duval S, Tweedie R: Trim and fill: a simple funnel-plot-based method of testing and adjusting for publication bias in meta-analysis. Biometrics 2000;56:455-463.

22 Orwin RG: A fail-safe $N$ for effect size in metaanalysis. J Educ Behav Stat 1983;8:157-159.
Shinaberger CS, Kopple JD, Kovesdy CP, et al: Ratio of paricalcitol dosage to serum parathyroid hormone level and survival in maintenance hemodialysis patients. Clin J Am Soc Nephrol 2008;3:1769-1776.

24 St Peter WL, Li S, Liu J, et al: Effects of monthly dose and regular dosing of intravenous active vitamin $\mathrm{D}$ use on mortality among patients undergoing hemodialysis. Pharmacotherapy 2009;29:154-164.

25 Kalantar-Zadeh K, Miller JE, Kovesdy CP, et al: Impact of race on hyperparathyroidism, mineral disarrays, administered vitamin D and survival in hemodialysis patients. J Bone Miner Res 2010;25:2724-2734.

26 Jean G, Lataillade D, Genet L, et al: Impact of hypovitaminosis D and alfacalcidol therapy on survival of hemodialysis patients: results from the French ARNOS study. Nephron Clin Pract 2011;118:c204-c210.

27 Kalantar-Zadeh K, Kuwae N, Regidor DL, et al: Survival predictability of time-varying indicators of bone disease in maintenance hemodialysis patients. Kidney Int 2006;70:771780.

28 Lee GH, Benner D, Regidor DL, Kalantar-Zadeh K: Impact of kidney bone disease and its management on survival of patients on dialysis. J Ren Nutr 2007;17:38-44.

29 Kovesdy CP, Ahmadzadeh S, Anderson JE, Kalantar-Zadeh K: Association of activated vitamin $\mathrm{D}$ treatment and mortality in chronic kidney disease. Arch Intern Med 2008;168: 397-403.

30 Marco MP, Craver L, Betriu A, et al: Higher impact of mineral metabolism on cardiovascular mortality in a European hemodialysis population. Kidney Int Suppl 2003;63:S111S114.

31 Melamed ML, Eustace JA, Plantinga L, et al: Changes in serum calcium, phosphate, and PTH and the risk of death in incident dialysis patients: a longitudinal study. Kidney Int 2006; 70:351-357.

-32 Naves-Díaz M, Alvarez-Hernández D, Passlick-Deetjen J, et al: Oral active vitamin D is associated with improved survival in hemodialysis patients. Kidney Int 2008;74:10701078.

33 Shoben AB, Rudser KD, de Boer IH, Young B, Kestenbaum B: Association of oral calcitriol with improved survival in nondialyzed CKD. J Am Soc Nephrol 2008;19:1613-1619.

34 Shoji T, Shinohara K, Kimoto E, et al: Lower risk for cardiovascular mortality in oral 1a-hydroxy vitamin D3 users in a haemodialysis population. Nephrol Dial Transplant 2004; 19:179-184.

- 35 Sugiura S, Inaguma D, Kitagawa A, et al: Administration of alfacalcidol for patients with predialysis chronic kidney disease may reduce cardiovascular disease events. Clin Exp Nephrol 2010;14:43-50.
36 Taniguchi M, Yamada S, Tokumoto M, Tsuruya $\mathrm{K}$, Hirakata $\mathrm{HN}$ : Active vitamin D therapy is associated with lower mortality, especially in low 25(OH)D levels. Renal Week 2010, Abstract Sessions, 2010 TH-PO199. http://www.asn-online.org/education_and_ meetings/kidneyweek/archives/RW10Abstracts.pdf (accessed January 25, 2012).

37 Teng M, Wolf M, Ofsthun MN, et al: Activated injectable vitamin $\mathrm{D}$ and hemodialysis survival: a historical cohort study. J Am Soc Nephrol 2005;16:1115-1125.

38 Tentori F, Hunt WC, Stidley CA, et al: Mortality risk among hemodialysis patients receiving different vitamin $\mathrm{D}$ analogs. Kidney Int 2006;70:1858-1865.

39 Tentori F, Albert JM, Young EW, et al: The survival advantage for haemodialysis patients taking vitamin $\mathrm{D}$ is questioned: findings from the Dialysis Outcomes and Practice Patterns Study. Nephrol Dial Transplant 2009;24:963-972.

40 Wolf M, Betancourt J, Chang Y, et al: Impact of activated vitamin $\mathrm{D}$ and race on survival among hemodialysis patients. J Am Soc Nephrol 2008;19:1379-1388.

41 Olgaard K, Lewin E: Use (or misuse) of vitamin $\mathrm{D}$ treatment in $\mathrm{CKD}$ and dialysis patients. Nephrol Dial Transplant 2008;23: 1786-1789.

42 Palmer SC, McGregor DO, Craig JC, et al: Vitamin $\mathrm{D}$ compounds for people with chronic kidney disease not requiring dialysis. Cochrane Database Syst Rev 2009;CD008175.

43 Palmer SC, McGregor DO, Craig JC, et al: Vitamin D compounds for people with chronic kidney disease requiring dialysis. Cochrane Database Syst Rev 2009;CD005633.

44 Block GA, Klassen PS, Lazarus JM, et al: Mineral metabolism, mortality, and morbidity in maintenance hemodialysis. J Am Soc Nephrol 2004; 15:2208-2218.

45 Cheng J, Zhang W, Zhang X, Li X, Chen J: Efficacy and safety of paricalcitol therapy for chronic kidney disease: a meta-analysis. Clin J Am Soc Nephrol 2012;7:391-400.

46 Reeves BC, Deeks JJ, Higgins JPT, Wells GA: Chapter 13: Including non-randomized studies; in Higgins JPT, Green S (eds): Cochrane Handbook for Systematic Reviews of Interventions. Version 5.0.1. Chichester, The Cochrane Collaboration, John Wiley \& Sons Ltd., 2008, pp 391-432.

-47 Cox E, Martin BC, Van Staa T, et al: Good research practices for comparative effectiveness research: approaches to mitigate bias and confounding in the design of nonrandomized studies of treatment effects using secondary data sources. Value Health 2009;12:1053-1061.

48 EVOLVE Trial Investigators: Effect of cinacalcet on cardiovascular disease in patients undergoing dialysis. New Engl J Med 2012; 367:2482-2494.

49 Thadhani R, Appelbaum E, Pritchett Y, et al: Vitamin D therapy and cardiac structure and function in patients with chronic kidney disease: the PRIMO randomized controlled trial. JAMA 2012;307:674-684. 